Trauma Berufskrankh 2016 · [Suppl 2]:

18:S101-S104

DOI 10.1007/s10039-016-0126-9

Online publiziert: 22. Januar 2016

(c) Springer-Verlag Berlin Heidelberg 2016
D. Schneidmueller · V. Bühren

Abteilung für Unfallchirurgie, Sportorthopädie und Kindertraumatologie,

Klinikum Garmisch-Partenkirchen, BG-Unfallklinik Murnau

\section{Verletzungen des Jugendlichen - die Übergangsfraktur}

\section{Nomenklatur, Diagnostik und Therapie}

Je nach Reife des Kindes kommt es in einem spezifischen Alter durch hormonelle und humorale Einflüsse zu einem langsamen Verschluss der Wachstumsfugen. Hieraus resultiert eine Veränderung des Verletzungsmusters. Durch den bereits verknöcherten Anteil der Wachstumsfuge werden einwirkende Kräfte zum Gelenk hin abgeleitet, sodass es je nach Reifezustand der Fuge zu einem Ausbrechen eines mehr oder weniger großen epiphysären Fragments kommt. Diese sog. Übergangsfraktur kann grundsätzlich an jeder Lokalisation auftreten. Am häufigsten beobachtet man sie an der distalen Tibia mit einer Inzidenz von $11 \%$ aller Sprunggelenkfrakturen, weshalb sich dieser Beitrag im Weiteren auf diese Lokalisation beschränkt [4].

\section{Frakturmorphologie/ Klassifikation}

Am Sprunggelenk beginnt die Wachstumsfuge sich um das 12. Lebensjahr über einen Zeitraum von 18 Monaten von medial über den dorsalen Anteil zu verschließen, zuletzt mineralisiert somit der ventrolaterale Anteil der Fuge (• Abb. 1, $[2,3])$. Hierdurch kommt es zu einer Veränderung des Frakturverlaufs in der Grenzzone des Mineralisationsvorgangs,

Es handelt sich um eine Übernahme des Beitrages 10.1007/s10039-014-2119-x. wodurch je nach aktuell vorherrschender Phase des Fugenverschlusses ein mehr oder weniger großes ventrolaterales epiphysäres Fragment ausbricht. Kurz vor dem vollständigen Fugenschluss entspricht diese Fraktur einem knöchernen Syndesmosenausriss. Wirken zusätzliche Torsionskräfte ein, kann analog zum hinteren Volkmann-Dreieck beim Erwachsenen zusätzlich zum ventrolateralen epiphysären Bruch eine dorsale Fraktur auftreten.

Abhängig von der Anzahl der Fragmente werden die Übergangsfrakturen weiter differenziert $[6,9]$. Ihnen allen gemeinsam ist die ventrolaterale epiphysäre Fraktur. Liegt nur diese vor, spricht man von der sog. Twoplane-Fraktur oder auch, nach dem Erstbeschreiber, einer Tillaux-Fraktur (- Abb. 2). Besteht zusätzlich eine dorsale metaphysäre Fraktur, spricht man von der sog. Triplane-IFraktur (- Abb. 3a). Verläuft diese dorsale Fraktur durch Epi- und Metaphyse, handelt es sich um eine Triplane-II-Fraktur (- Abb. 3b).

\section{Diagnostik}

Die Diagnose einer Übergangsfraktur kann in aller Regel bereits am konventionellen Röntgenbild gestellt werden.

Eine genaue Differenzierung zwischen Twoplane-, Triplane-I- und -II-Frakturen ist oft nur anhand einer Schnittbild- gebung möglich [10, 11]. Sie wird v. a. zur Beurteilung des dorsalen Frakturabschnitts und bei vermeintlich undislozierten Übergangsfrakturen empfohlen. Die beste Beurteilung einer Dislokation lässt sich dabei durch eine Computertomografie erreichen $[5,12]$.

\section{Therapie}

Entsprechend den Richtlinien einer jeden Gelenkfraktur ist das Ziel, eine anatomische Gelenkrekonstruktion zu erreichen und möglichst eine Kompressionsosteosynthese nach Reposition durchzuführen.

Undislozierte Frakturen (2 mm Dehiszenz, keine Gelenkstufe) können konservativ im Unterschenkelgips für 4 bis 6 Wochen therapiert werden. Dislozierte Frakturen müssen anatomisch unter Anästhesie reponiert werden. $\mathrm{Ob}$ eine geschlossene Reposition erfolgen kann oder eine offene Darstellung der Frakturregion notwendig ist, wird kontrovers diskutiert [6, 7]. Im Zweifel sollte die Frakturreposition nach Meinung der Autoren über einen kleinen ventralen Zugang kontrolliert werden.

Aufgrund des veränderten Frakturmusters ist eine Verschraubung der epiphysären Fraktur von medial schwierig. Eine optimale Kompression erreicht man durch eine direkte, schräg von anterolateral nach dorsomedial verlaufende Schraubenosteosynthese. 


\section{Typische Frakturen und Luxationen}

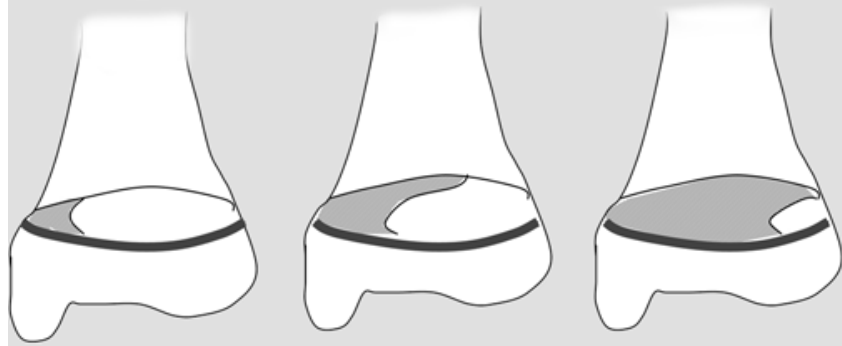

Abb. $1 \Delta$ Fugenverschluss an der distalen Tibia, Beginn medial, über dorsal nach ventrolateral (grau bereits verschlossener Fugenanteil)

Abb. 2 Twoplane-Fraktur: ventrolaterale epiphysäre Fraktur. (Aus [8])

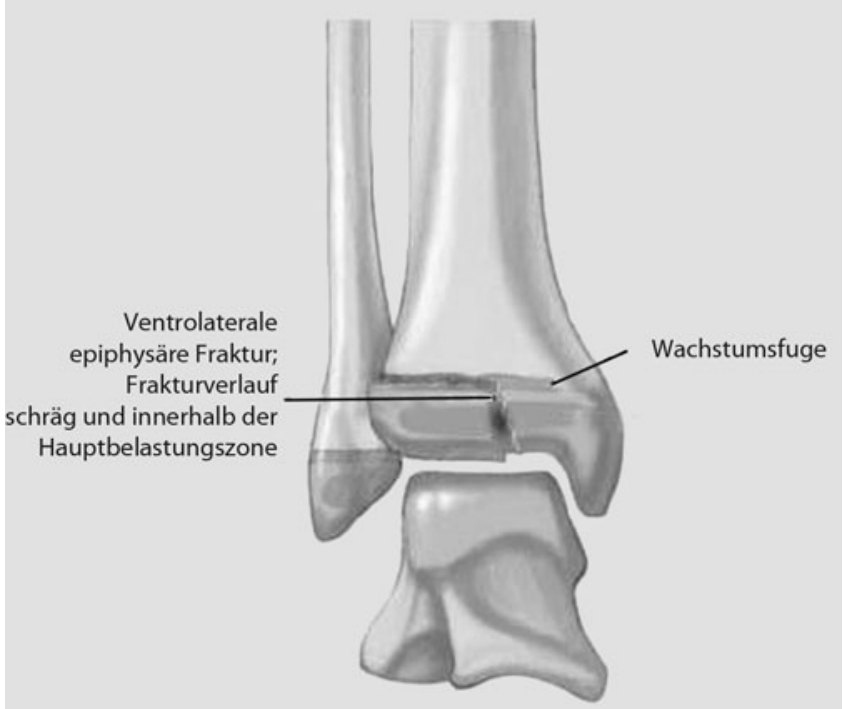

Abb. $3<$ Triplane-Fraktur, a Triplane I: ventrolaterale epiphysäre + dorsale metaphysäre Fraktur, b Triplane Il: ventrolaterale epiphysäre + dorsale epimetaphysäre Fraktur. (Aus [8])
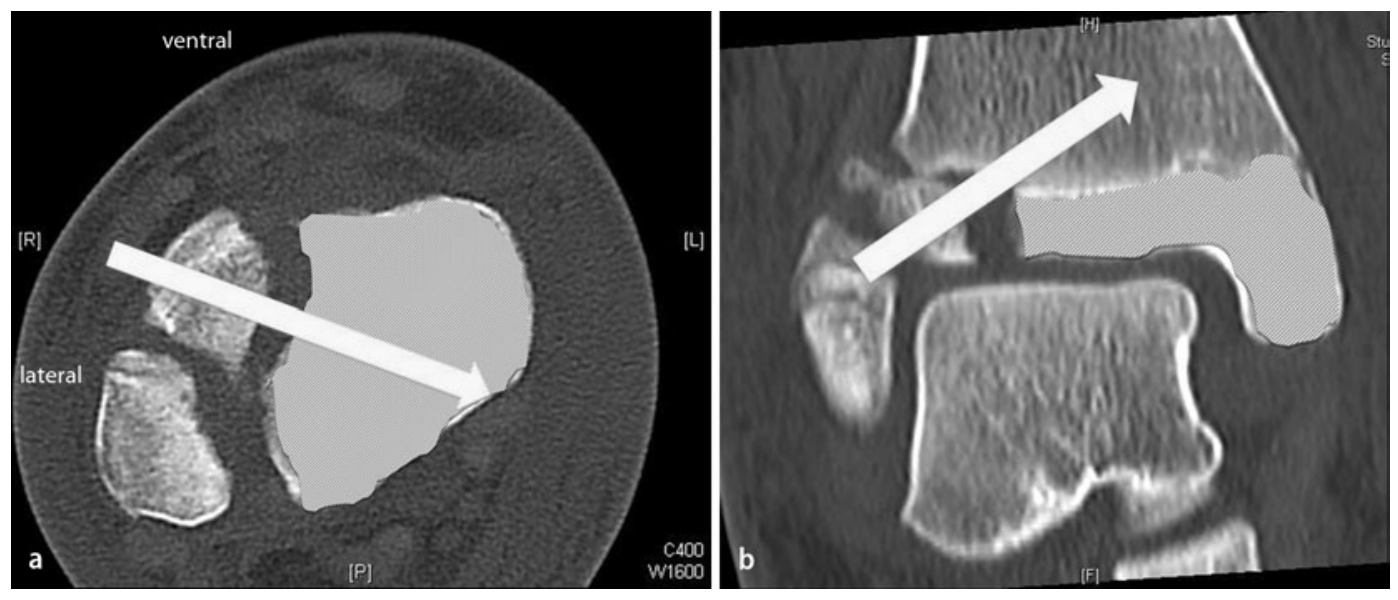

Abb. $4<$ Schraubenosteosynthese der TwoplaneFraktur, gleichmäßig grau gefärbte Fläche bereits verknöcherter Fugenanteil, Schraubenverlauf (Pfeil) a in der axialen, $\mathbf{b}$ in der koronaren Schicht 


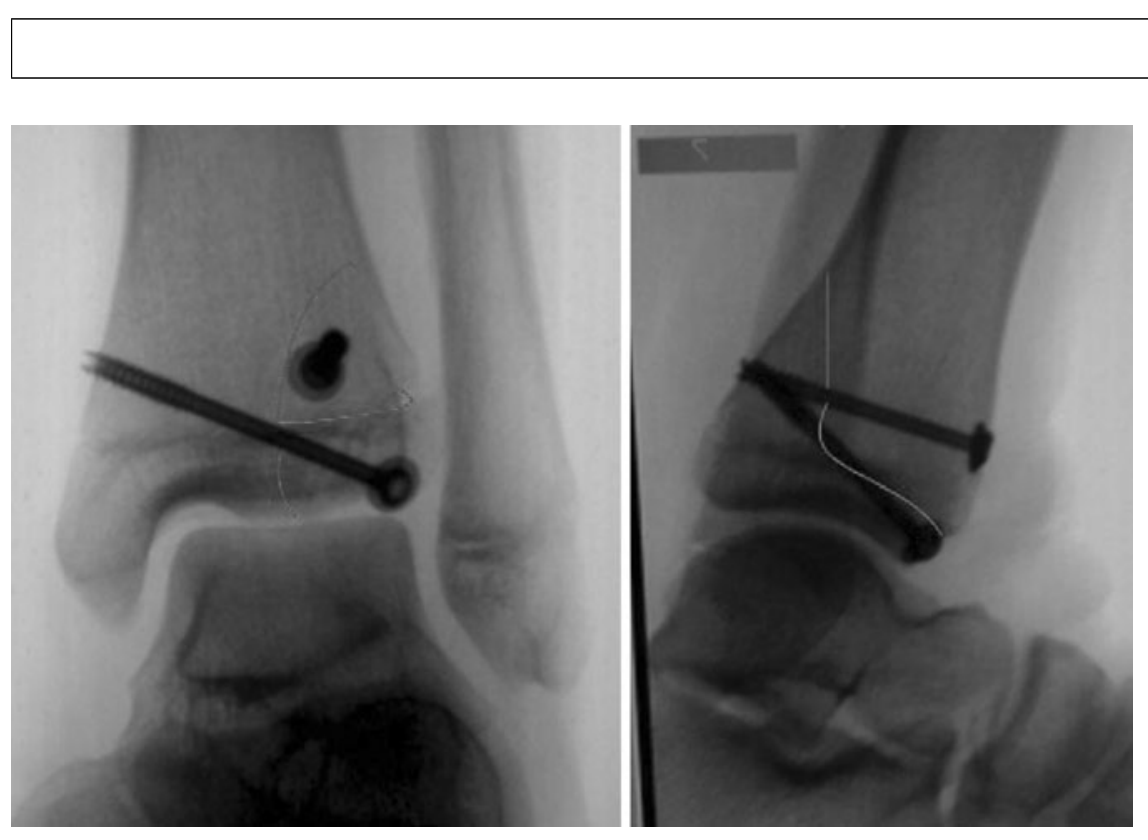

Abb. $5 \Delta$ Osteosynthesetechnik einer Triplane-I-Fraktur, direkte Verschraubung der ventrolateralen epiphysären und indirekte Verschraubung der dorsalen Fraktur

Dabei kann die (bereits verschlossene) Wachstumsfuge gekreuzt werden, ohne dass Wachstumsstörungen zu befürchten wären (• Abb.4). Die Verschraubung des dorsalen Frakturanteils gelingt in der Regel indirekt geschlossen von ventral, analog zur Versorgung des hinteren Volkmann-Dreiecks (• Abb. 5).

\section{Prognose}

Aufgrund des bereits begonnen Fugenschlusses sind im Gegensatz zu den fugennahen Verletzungen bei noch weit offenen Wachstumsfugen (Salter und Harris I-IV) keine relevanten Wachstumsstörungen mehr zu befürchten. Wie nach allen Gelenkfrakturen ist jedoch, v. a. bei unzureichender Reposition, mit Spätfolgen im Sinne einer Früharthrose zu rechnen [1]. Im Vergleich zur Salter- und Harris-III- und -IV-Verletzungen, bei denen die Fraktur immer sehr weit medial liegt, rückt diese bei der Übergangsfraktur je nach Reifezustand immer weiter nach lateral in die Hauptbelastungszone, was zumindest theoretisch ein größeres Risiko für eine Früharthrose darstellt. Vergleichende Untersuchungen mit einer ausreichenden Fallzahl und einem entsprechenden Nachuntersuchungszeitraum existieren jedoch nicht.

\section{Fazit für die Praxis}

- Die Übergangsfraktur stellt die typische Gelenkfraktur des Jugendlichen bei bereits begonnenem Fugenschluss dar.

- Die Übergangsfraktur weist ein stereotypes Frakturmuster auf, welches sich von den Verletzungen bei weit offener Fuge unterscheidet.

- Diagnostik und Therapie der Übergangsfraktur müssen an die veränderten Verhältnisse angepasst werden.

- Ziel der Behandlung ist eine anatomische Gelenkrekonstruktion, um eine Früharthrose zu vermeiden.

\section{Korrespondenzadresse}

\section{PD Dr. D. Schneidmueller}

Abteilung für Unfallchirurgie, Sportorthopädie und Kindertraumatologie, Klinikum GarmischPartenkirchen, BG-Unfallklinik Murnau, Prof.-Küntscher-Straße 8, 82418 Murnau dorien.schneidmueller@bgu-murnau.de

\section{Einhaltung ethischer Richtlinien}

Interessenkonflikt. D. Schneidmueller und V . Bühren geben an, dass kein Interessenkonflikt besteht.

Dieser Beitrag beinhaltet keine Studien an Menschen oder Tieren.

The supplement containing this article is not sponsored by industry.
Trauma Berufskrankh 2016 · [Suppl 2]: 18:S101-

S104 DOI 10.1007/s10039-016-0126-9

๑) Springer-Verlag Berlin Heidelberg 2016

D. Schneidmueller $\cdot$ V. Bühren

Verletzungen des Jugendlichen - die Übergangsfraktur. Nomenklatur, Diagnostik und Therapie

\section{Zusammenfassung}

Definition. Die Übergangsfraktur stellt die typische Gelenkverletzung des Jugendlichen zum Zeitpunkt des Fugenschlusses dar. Durch die teilweise verknöcherte Wachstumsfuge kommt es zu einer Veränderung des beim Kind immer stereotypen Frakturverlaufs. Es entsteht ein spezifisches Frakturmuster, welches je nach Anzahl der Fragmente in Two- und Triplane-Frakturen unterschieden wird.

Diagnostik. Zur genauen Darstellung aller Frakturanteile spielt neben dem konventionellen Röntgenbild die Schnittbildgebung eine maßgebliche Rolle.

Behandlung. Ziel der Therapie ist eine anatomische Gelenkrekonstruktion, um die Spätfolge Früharthrose zu vermeiden.

\section{Schlüsselwörter}

Sprunggelenkverletzungen .

Knochenbrüche - Twoplane - Triplane ·

Jugendliche

\section{Injuries in adolescents - transitional fractures. Nomenclature, diagnosis and treatment}

\begin{abstract}
Definition. The transitional fracture represents the typical joint injury of adolescents in the period of growth plate closure. The partially ossified growth plate leads to a change in the stereotype fracture pattern in children. The result is a specific fracture pattern which can be classified into two- and triplane fractures, according to the number of fragments. Diagnosis. Conventional radiographs play a major role in diagnosis, whereby cross-sectional imaging helps to distinguish between the specific fracture types.

Treatment. The goal of therapy is anatomical joint reconstruction to avoid long-term consequences of early osteoarthritis.
\end{abstract}

\section{Keywords}

Ankle injuries · Bone fractures · Twoplane .

Triplane $\cdot$ Adolescents 


\section{Literatur}

1. Cooperman DR, Spiegel PG, Laros GS (1978) Tibial fractures involving the ankle in children. The socalled triplane epiphyseal fracture. J Bone Joint Surg Am 60:1040-1046

2. Ertl JP, Barrack RL, Alexander AH et al (1988) Triplane fracture of the distal tibial epiphysis: long term follow up. J Bone Joint Surg Am 70:967-976

3. Kärrholm J (1997) The triplane fracture: four years of follow-up of 21 cases and review of the literature. J Pediatr Orthop B 6:91-102

4. Krauss R, Schneidmüller D, Röder C (2005) Häufigkeit von Frakturen der langen Röhrenknochen im Wachstumsalter. Dtsch Arztebl 102:A-838/B708/C-661

5. Laarhoven CJ van, Severijnen RS, Werken C van der (1995) Triplane fractures of the distal tibia. J Foot Ankle Surg 34:556-559

6. Laer $L$ von (2001) Frakturen und Luxationen im Wachstumsalter, 4. Aufl. Thieme, Stuttgart New York, S 383

7. Peiro A, Aracil J, Martos F et al (1981) Triplane distal tibial epiphyseal fracture. Clin Orthop Relat Res 160:196-200

8. Schneidmüller D, Marzi I (2008) Die operative Behandlung von Übergangsfrakturen der distalen Tibia. Oper Orthop Trauma 20:254-263

9. Schneidmüller D, Marzi I (2009) Sprunggelenk. In: Marzi I (Hrsg) Kindertraumatologie, 2. Aufl. Springer, Berlin Heidelberg New York

10. Schneidmueller D, Sander AL, Wertenbroek M et al (2014) Triplane fractures: do we need cross-sectional imaging? Eur J Trauma Emerg Surg 40:37-43

11. Seifert J, Matthes G, Hinz P et al (2003) Role of magnetic resonance imaging in the diagnosis of distal tibia fractures in adolescents. J Pediatr Orthop 23:727-732

12. Spiegel PG, Mast JW, Cooperman D et al (1984) Triplane fractures of the distal tibial epiphysis. Clin Orthop Relat Res 188:74-89

\section{Call for Papers: Herbert-Lauter- bach-Preis 2016}

\section{BG Kliniken veranstalten 15.000-Euro-} Wissenschaftswettbewerb

Mit dem Herbert-Lauterbach-Preis würdigen die BG Kliniken auch 2016 wieder herausragende wissenschaftliche Leistungen auf den Gebieten Unfallmedizin und Berufskrankheiten. Im Fokus stehen dabei Themen, die für die medizinische Versorgung von Versicherten der Berufsgenossenschaften und Unfallkassen eine Rolle spielen, wie z.B. typische Verletzungen bei Arbeitsunfällen.

Bewerberinnen und Bewerber um den mit 15.000 Euro dotierten Preis sollten ihre Studien- oder Forschungsarbeiten im Kalenderjahr 2015 entweder in einer Fachpublikation veröffentlicht oder als Manuskript niedergelegt haben. $\mathrm{Zu}$ den erforderlichen Bewerbungsunterlagen gehören vier Druckexemplare der Arbeit in deutscher Sprache, eine kurze Zusammenfassung sowie eine eidesstattliche Erklärung, dass die Arbeit nicht bereits anderweitig ausgezeichnet oder zeitgleich einem weiteren Prüfungsgremium vorgelegt wurde. Die Unterlagen können jedoch auch per E-Mail an HYPERLINK "mailto:forschung@bg-kliniken. de" forschung@bg-kliniken.de oder über das Onlinebewerbungsformular unter HYPERLINK "http://www.bg-kliniken.de/ akutmedizin-rehamedizin/forschung/ herbert-lauterbach-preis/" www.bg-kliniken. de/akutmedizin-rehamedizin/forschung/ herbert-lauterbach-preis/ eingereicht werden.

\section{Bewerbungsschluss ist der 30. April \\ 2016.}

Bewerbungen per Post senden Sie bitte an:

BG Kliniken - Klinikverbund der gesetzlichen Unfallversicherung gGmbH Stichwort "Herbert-Lauterbach-Preis" Mittelstraße 51

10117 Berlin 